\title{
Tangence
}

\section{L'héritage œcuménique}

\section{Lecture d'une mosaiique religieuse}

\section{Manon Lewis}

Numéro 41, octobre 1993

Interdiscurtivité dans l'œuvre de Victor-Lévy Beaulieu

URI : https://id.erudit.org/iderudit/025780ar

DOI : https://doi.org/10.7202/025780ar

Aller au sommaire du numéro

Éditeur(s)

Tangence

ISSN

0226-9554 (imprimé)

1710-0305 (numérique)

Découvrir la revue

Citer cet article

Lewis, M. (1993). L'héritage œcuménique : lecture d'une mosaïque religieuse.

Tangence, (41), 95-111. https://doi.org/10.7202/025780ar d'utilisation que vous pouvez consulter en ligne.

https://apropos.erudit.org/fr/usagers/politique-dutilisation/ 


\title{
L'héritage œcuménique. Lecture d'une mosaïque religieuse
}

\section{Manon Lewis *}

\begin{abstract}
Aucun pays, de toute façon, ne s'habite autrement que dans l'usurpation: on ne devrait pas y être et on s'y trouve, et on n'en est pas parti qu'on voudrait déjà y revenir, tel le peuple juif allant de l'un à l'autre, minorité par l'esprit. Et l'esprit étant aux antipodes mêmes de la réconciliation, voilà bien où il faut vivre jusqu'à sa mort, dans cet entre-deux fait de feu et d'eau, en même temps incendie et incendiaire, noyade et noyé puisque si la Loi existe, les hommes n'ont pas suffisamment de présence donc de force, pour être à sa hauteur, dans la blancheur qui la constitue.
\end{abstract}

Victor-Lévy Beaulieu, L'béritage

Véritable raz-de-marée téléromanesque pendant trois saisons $^{1}$, L'béritage de Victor-Lévy Beaulieu apparaît telle une fresque où s'enchevêtrent les croyances les plus diverses. Le vaudou, le bouddhisme zen et le paganisme côtoient en effet le protestantisme notoire d'un Xavier Galarneau. Aux récits bibliques se mêlent encore les mythologies amérindienne et grecque. De plus, à travers la guerre de succession à laquelle se

Manon Lewis est étudiante au doctorat en Sciences des religions à l'Université du Québec à Montréal.

1 Les quatre-vingt-six épisodes du téléroman, diffusés sur les ondes de Radio-Canada de l'automne 1987 au printemps 1990, ont connu un succès retentissant. Les sondages confirment la première place de L'béritage parmi les émissions les plus regardées avec, certaines semaines, quelque deux millions de spectateurs et spectatrices. ( $C f$. Louise Cousineau, "L'héritage reprend la tête du peleton", dans $L a$ Presse, samedi 4 novembre 1989). 
96

livre impitoyablement la tribu des Galarneau, c'est à une étonnante histoire sainte où perce l'antique sagesse grecque que convie le téléromancier.

Il s'agira, dans un premier temps, de souligner la présence des nombreuses trames religieuses qui tissent le texte de L'béritage. Puis, en second lieu, par le recours à l'herméneutique, certaines balises seront posées au fil de ce téléroman-fleuve. Il convient toutefois de rappeler - de rameuter - les grandes lignes de cette saga beaulieusienne.

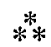

L'béritage, c'est d'abord l'histoire d'un inceste: celui que commet, quatorze ans avant le début du récit, Xavier Galarneau avec sa fille aînée, Miriam. Depuis cette nuit fatale où il a dérogé à la Loi, Xavier vit avec ce secret douloureux. Afin que l'univers entier ne bascule pas, l'austère protestant de Trois-Pistoles garde fermement sa famille sous son emprise.

Le vieux roi, en effet, entend conserver son pouvoir et ses biens, ne serait-ce que pour les soustraire à son fils aîné et héritier présomptif, Miville, plus près du bouc émissaire que du dauphin. Le cadet, Junior, le fils bien-aimé, ne craint pas - au contraire de l'aîné - de se mesurer au père, de lui tenir tête. Julie, la benjamine, se contente pour sa part d'exercer son rôle de ménagère et de médiatrice. Frère puîné de Xavier et hommecheval dérisoirement attelé à une petite voiture, Gabriel subit également le joug du souverain tyrannique.

À des centaines de kilomètres en amont du fleuve, Miriam partage sa vie, depuis quatorze ans, entre son travail et son fils Maxime, fruit de l'inceste. Seul Philippe Couture, patron de la petite entreprise d'éditions où est employée Miriam, connaît l'existence de l'enfant.

Un fragile ordre des choses règne d'un bord et de l'autre du fleuve, jusqu'à l'arrivée à la ville de Stéphanie - fille de Gabriel Galarneau et donc cousine germaine de Miriam. Stéphanie instaure, malgré elle, une ronde d'allers et de retours incessants entre Trois-Pistoles et Montréal. Ainsi Albertine, sa mère, scert cie son lit conjugal, quitte livres et mari et gagne la métropole, révélant du même coup l'adresse de Miriam à sor beau-frère 
Xavier et attirant sur elle-même la passion amoureuse de Philippe, qui est aussi poète à ses heures. Les liens se tissent lentement entre les habitants du haut et du bas du fleuve. Des rencontres se nouent, des alliances et des conflits surgissent, des pactes se font et se défont. Des frontières s'estompent et des secrets se dévoilent.

Loin d'apaiser les tensions, la mort de Xavier vient, à l'inverse, intensifier les luttes familiales. Miriam prend la relève du père dans cette guerre de succession qui l'oppose à Miville. Les affrontements se poursuivent sous les feux de l'été pistolois et s'achèvent par l'incendie de l'Hôtel de la Gare. Philippe Couture et quatre femmes y périssent.

À l'éclatement et à l'embrasement du monde succède sa reconstruction. Un nouvel hôtel s'élevera bientôt sur l'emplacement de l'ancien. Les funérailles font place aux épousailles ${ }^{2}$ qui sont l'occasion d'une vaste réconciliation. Miville, seul, hérite de la terre et de la maison paternelles. Miriam repart, comme quinze ans auparavant, héritière et héraut de la Loi. Junior et Julie poursuivent le rêve du père, celui des courses de chevaux, et perpétuent en outre le modèle originel du couple incestueux - sans toutefois que cette relation ne génère le malheur, à la différence du premier. Plongée dans l'écriture, Albertine continue pour sa part l'œuvre de Philippe Couture, c'est-à-dire raccommoder l'univers au fil des mots.

\section{D'une foi à l'autre}

Fait relativement peu connu, au tournant du siècle dernier, le Bas-du-Fleuve est le siège d'une importante expansion presbytérienne $^{3}$. L'influence d'un Charles-Pascal Chiniquy y est pour

2 Il s'agit de triple noces, celles de Gabriel Galanneau et Blanche Soucy (veuve et mère vertueuse), de Stéphanie Galarneau et Florent Corbin (jeune gérant de la caisse populaire de Trois-Pistoles), et de Ti-Bob Cayouette et Judith Martin (amoureux qu'éconduisent respectivement Julie et Junior).

3 Cette église, fondée sur la doctrine de Calvin, "a développé un système ecclésiastique qui donne le gouvernement ecclésial à un corps mixte, composé de pasteurs et de laiquesn. Aubert April, Emmanuel Rioux et al., Saint-Cyprien de Rivière-du-Loup. Un siècle de labeur et de progrès!, Rimouski, (C) Les Amis de Hocquart, 1987 [1986], p. 225, note 223, passim. Cf. plus particulièrement le chapitre intitulé "Histoire d'un schisme appréhendé ", d'Aubert April, p. 210-218. 
beaucoup. Natif de la région, "grand apôtre de la tempérance" et ancien prêtre catholique, Chiniquy se joint en 1859 à l'Église presbytérienne. Ses coreligionnaires parcourent les campagnes "à l'affût des situations qui pourraient favoriser leur incursion dans des milieux qui leur semblaient réfractaires jusque-là " ${ }^{4}$. Mécontents de ce que l'Église catholique leur refuse les services d'une église et d'un prêtre permanent, quelques habitants de SaintCyprien par exemple voient d'un bon ceil l'arrivée de ces ministres. Profitant de l'occasion, les missionnaires presbytériens s'installent et construisent une petite chapelle, ralliant ainsi quelques adeptes, non sans provoquer de vives querelles au sein de la petite communauté 5 . Quelques années plus tard, faute de fidèles, la mission est abandonnée.

S'inspirant librement de certains de ces faits historiques, Victor-Lévy Beaulieu attribue une origine huguenote aux Galarneau: «Les Galarneau descendent de protestants français qui, à la fin du siècle dernier, avaient même ouvert une mitaine dans le bas du Fleuve." $\left(23,17^{6}\right)$ Refoulés de Saint-Cyprien où les catholiques ont incendié leur "mitaine" à peine bâtie ${ }^{7}$, le grand-père de Xavier et sa famille "se sont r'tranchés dans leurs terres avec leur Bible" $(23,18)$. Exilés à Trois-Pistoles donc, ils subissent encore là l'animosité de la population. Xavier lui-même est longtemps mis au ban de ce "village qui voulait pas oublier qu'son

4 Ibid., p. 211. Un certain Moïse Abram, étudiant en théologie, est de ceux-là. Beaulieu emprunte le nom pour créer ce Moïse Abraham, petitfils en quête de son homonyme jadis précepteur chez les Galarneau.

5 L'écrivain Adrien Thério, né à Saint-Cyprien - situé à quelque trente kilomètres au sud de Trois-Pistoles - narre cet épisode de dissension des résidents du chemin Taché dans son roman La colère du père, Montréal, Éditions Jumonville, 1974. Ce père, Gaudiose, partage par ailleurs avec Xavier Galarneau la même dureté et la même autorité tyrannique.

6 Les diverses références inscrites ainsi dans le corps du texte renvoient aux scénarios et dialogues du téléroman L'béritage. Le premier nombre indique l'épisode dont il est question, alors que le second marque la page d'où est tirée la citation ou l'information.

7 "Ce terme "mitaine" est une déformation de l'appellation anglaise "Meeting House", qui signifie "Temple"." April et al., op. cit., p. 225, note 232. L'incendie de la "mitaine" de Saint-Cyprien a bel et bien eu lieu mais plusieurs années après son abandon, non pas allumé par d'ardents défenseurs de foi catholique, mais plutôt par un garçonnet soucieux de faire enfin disparaître "le monument de la discorde" de ses aînés (ibid., p. 218). 
père était protestant. $(23,19)$. Cette originalité religieuse, par rapport à une majorité québécoise catholique, pose d'emblée le caractère marginal - et pour ainsi dire déjà sacré (du latin sacer, c'est-à-dire "séparé") - de la tradition familiale que perpétue Xavier Galarneau.

À travers maintes pratiques ascétiques telles le jeûne dominical, les méditations quotidiennes ou la retraite qu'il s'impose au déclin de l'hiver, Xavier se soumet à la Loi divine. "La Loi est faite comme est faite... pis y a pas moyen d'passer à côté" $(18,50)$, "C'pas moi qui l'veux: c'est la Loi!..." $(37,19)$, scande-t-il invariablement.

Face à la rigidité du légalisme paternel se déploie une forme de paganisme débridé, d' "effervescence dionysiaque " 8 dont le fils cadet des Galarneau, Junior, se révèle le plus illustre représentant et l'Hôtel de la Gare, le principal site cultuel. L'hôtel perpétue en effet nombre de fêtes païennes. L'une d'elles, l'Halloween, donne lieu à une étrange mascarade réunissant démons et merveilles, ivresse et excès de toutes sortes?

L'Hôtel de la gare est à nouveau le théâtre de festivités autour, cette fois, d'une épluchette de blé d'Inde. Maître de cérémonie déguisé pour la circonstance en épi de maïs, Ti-Bob Cayouette ${ }^{10}$ rappelle que "les Aztèques [...] prétendaient que la vie a commencé avec un épi de blé d'inde ${ }^{11}{ }^{11}(60,66)$ Si la culture du

8 Cf. e.g., Michel Maffesoli, L'ombre de Dionysos. Contribution à une sociologie de l'orgie, Paris, Méridiens/Anthropos, coll. "Sociologies au quotidien ", 1982, 212 p. Dieu de la nuit, de l'ivresse, des sens, du chaos et de l'excès, Dionysos paraît très proche d'un Junior Galarneau affirmant au lendemain d'une soirée généreusement arrosée: "C'qui m'intéresse, c'pas qu'ça mène quéque part mais que ça s'démène de toutes parts.... $(6,7)$

9 Lointaine survivance de la Samain, qui célébrait le premier de l'an celtique, cette fête "hors du temps et du monde réels" permettait aux créatures de l'au-delà d'envahir l'univers des humains. Cette unité des deux mondes était en outre "prétexte à beuveries et à festins". Si l'Halloween n'est plus célébré sous l'égide des druides, c'est toutefois sous la surveillance de Maggie Cayouette la propriétaire de l'hôtel, digne descendante des Celtes, costumée pour l'occasion en "sorcière irlandaise $(9,76)$ que se déroulent les réjouissances. (Cf. Françoise Le Roux, "La religion des Celtes", dans Henri-Charles Puech (dir.), Histoire des religions, tome I, Paris, Gallimard, coll. "Encyclopédie de la Pléiade", 1970 , p. 815.$)$

10 Neveu de Maggie l'hôtelière et soupirant bafoué de Julie Galarneau.

11 Gabriel enchaîne: "J'sais pas si l'monde a effectiv'ment commencé avec un épi d'blé d'inde... comme le prétend Ti-Bob. Moi, j'ai plutôt l'impression 
100

maïs apparaît centrale pour plusieurs populations nordaméricaines, des rituels agraires des quatre coins du monde soulignent par ailleurs la période de récoltes diverses ${ }^{12}$. L'épluchette de blé d'Inde commémore à sa façon les traditionnelles fêtes collectives des moissons.

Victor-Lévy Beaulieu introduit également la troublante présence du vaudou à travers une jeune femme, Erzulie Maurice, native d'Haïti. Passionnément amoureuse du fils cadet des Galarneau, elle est de plus l'auteure et l'une des victimes du tragique incendie de l'Hôtel de la Gare.

Héritage des premiers esclaves amenés d'Afrique durant la deuxième moitié $\mathrm{du} \mathrm{xvII}^{\mathrm{e}}$ siècle, on sait que le vaudou haïtien s'est peu à peu constitué en une religion nationale et originale ${ }^{13}$. Au cours de nombreuses migrations, notamment aux États-Unis, "ce ne sont pas cependant les aspects bénéfiques du Vaudou religieux qui subsistent, écrit Roger Bastide, mais l'aspect de magie noire ". "C'est ce Vaudouisme abâtardi [...] par l'éloignement grandissant de ses origines " ${ }^{14}$ qui se donne essentiellement à voir dans le téléroman. Dans une "caricature du vaudou" (69, 70), Erzulie Maurice s'érige mambo (c'est-à-dire prêtresse) et se livre notamment à l'inévitable cliché de la poupée percée d'aiguilles. La jeune Haïtienne semble en outre connaître le sens complexe de son prénom: "Y a deux Erzulie dans l'monde vaudou... la vierge blanche qui est une sainte et la vierge noire qui a besoin du sacrifice de n'importe quel coq. " $(62,8)$ À la déesse blanche ou noire s'en ajoute une troisième, l'Erzulie rouge ${ }^{15}$ qui réclame vengeance et instaure le gigantesque sacrifice sanglant de l'hôtel.

qui devait y avoir une femme en d'sous de ça." $(60,67)$ Eliade écrivait en ce sens: "La solidarité reconnue entre la fécondité de la glèbe et celle de la femme constitue un des traits saillants des sociétés agricoles. "Mircea Eliade, Traité d'bistoire des religions, Paris, Petite Bibliothèque Payot, 1975 [1964], p. 221.

12 Cf. e.g., ibid., p. 294 et passim.

13 Cf. e.g., Roger Bastide, Les Amériques noires. Les civilisations africaines dans le nouveau monde, Paris, Payot, coll. "Bibliothèque scientifique", 1967, p. 144-155; Alfred Métraux, Le vaudou baitien, Paris, Gallimard, coll. "L'espèce humaine ", $6^{\mathrm{e}}$ édition, 1958, passim.

14 Bastide, op. cit., p. 153-154.

15 À propos de l'a Erzulie-zieux-rouges " ou "erzili-jé-rougen, voir Métraux, op. cit., p. 237 et Claude Planson, Le vaudou, Paris, M. A. Éditions, 1987, p. 105-106. 
Originaire de l'Inde - comme le vaudou haitien l'était du continent africain -, le bouddhisme au fil du temps et de ses migrations extrême-orientales a donné naissance à de nombreuses sectes. L'une d'elles, la "Doctrine du Coeur de Bouddha" (ou Bouddhahridaya), mieux connue sous le nom de zen, retient notre attention ${ }^{16}$. Éric Wisecomm ${ }^{17}$, qui a "troqué la philosophie occidentale pour le bouddhisme" (49, 49), devient en effet ambassadeur zen au pays des Galarneau. Recréant entre les quatre murs de sa chambre la sobriété des monastères japonais, Éric demeure fidèle au principe fondamental du zen, c'est-à-dire al'expérience vécue en sa simplicité première, dénouée de tout artifice" ${ }^{18}$.

D'autres personnages prêtent vie et visage à certaines mythologies et traditions. Albertine, par exemple, résume en quelque sorte le drame du peuple amérindien, des Malécites plus spécifiquement ${ }^{19}$. C'est un voyage vers le passé et la reconnaissance d'elle-même et de ses racines qu'entreprend Albertine à travers l'écriture. En même temps que se dénoue sa longue chevelure de princesse malécite, Albertine renoue avec ses souvenirs d'enfance: son père, le Bonhomme Fish ${ }^{20}$, vendeur itinérant qui, par

16 C.f. e.g., Daistz Teitaro Suzuki, Introduction au bouddbisme zen (trad. de l'anglais par Nicole Tisserand et Nicole Rougier), préface de C. G. Jung, Paris, Buchet/Chastel, 1978, passim.

17 Né en Belgique et professeur depuis peu au pensionnat que fréquente Maxime, le fils de Miriam, Wisecomm connaît une courte idylle avec la mère de son élève, avant de s'exiler en Amérique du Sud.

18 Ibid., p. 40.

19 Issus de la famille des Algonquins, les Malécites ont une étrange histoire qui refait surface depuis peu avec ses survivants oubliés qui avaient, semble-t-il, eux-mêmes désappris jusqu'à leur nom. Si bien disséminée, dispersée au Québec et aux États-Unis, il a fallu en effet près de 120 ans (l'année 1869 marque la cession - pour ne pas dire la dépossession de ses terres) pour que la tribu perdue se retrouve et renaisse dans les mémoires et dans son corps. Le 30 mai 1989, les Malécites sont reconnus officiellement comme onzième nation autochtone du Québec. En ce qui concerne les Malécites, $c f$. Robert Michaud (avec la collaboration de Gérard Filion), L'Isle-Verte vue du large, Montréal, Leméac, p. 25-37; Bruno Bisson, "Le Québec ne sera pas "redéfini" sans les Indiens", dans La Presse, samedi 11 août 1990, p. B-1 et B-3; Joseph-Charles Taché, Trois légendes de mon pays, Montréal, Librairie Beauchemin Limitée, 1912; April et al., op. cit., p. 12-15.

20 Découvert dans Le Saint-Laurent et ses îles de Damase Potvin, le Bonhomme Fish, "ce vieux pêcheur solitaire" ( $c f$. Victor-Lévy Beaulieu, Manuel de la petite littérature du Québec, Montréal, Éditions de l'Aurore, 
102

trop de désespoir, se tranche un jour la gorge; et ce Petit-Canada que jadis ils habitaient ${ }^{21}$. La cinquantaine révolue, Albertine renverse le temps pour ainsi atteindre l'an premier des choses. Un retour aux sources que couronne, à la fin du téléroman, son établissement à Cacouna, terre de ses ancêtres malécites ${ }^{22}$. Sous sa plume se concilient les mondes rouge et blanc.

Être hybride comme Albertine, sa compagne durant près de trente ans, Gabriel Galarneau participe aussi de deux univers. L'homme-cheval a en effet été initié, dès l'enfance, par le père d'Albertine, aux rituels de la chasse et de la pêche. De ses longues chevauchées aux quatre coins de Trois-Pistoles et de son imagination débridée s'échappent mille créatures chimériques, Magouas, Tarlanes qui peuplent les forêts et les cimetières protestants ${ }^{23}$.

Ici, la mythologie amérindienne rejoint celle des centaures de la Grèce ancienne par le biais de Gabriel. Confondant toutefois

1974 , p. 16), emprunte dans la fiction beaulieusienne, en plus de son nom, son "grand tablier d'cuir plein d'écailles de hareng pis son tomb'reau qu'y poussait d'vant lui " $(69,33-34)$.

21 "Le P'tit Canada, c'tait rien d'plusse qu'un amas d'cabanes où l'monde faisait pas aut'chose que d'vivoter. " $(64,7)$ De ce Petit-Canada, Abel Beauchemin, alter ego romanesque de Victor-Lévy Beaulieu, rappelle: "Dans mon enfance, je l'ai vécu aux Trois-Pistoles. Il y avait la paroisse Notre-Dame-des-Neiges et il y avait le Petit-Canada. Entre les deux, ce petit ruisseau qui partageait par ses eaux le monde d'en haut et le monde d'en bas. Dans le monde d'en haut, c'était la paroisse québécoise traditionnelle, avec toute son organisation hiérarchique: le curé, les notables, les commerçants et le peuple qui était sous leur sujétion. Dans le monde d'en bas, c'est-à-dire le Petit-Canada, on retrouvait les débris de la nation malécite, autrefois puissante et prospère en Gaspésie et dans le Bas-du-Fleuve. Ils vivaient dans des cabanes tout en démanche et étaient considérés comme des mécréants à qui il ne fallait même pas adresser la parole, de peur qu'ils ne nous contaminent." (Victor-Lévy Beaulieu, Docteur Ferron. Pèlerinage, Montréal, Stanké, 1991, p. 285.)

22 Au début du siècle, "le principal village maléchite [sic] occupe, en arrière des paroisses de Kakouna et de l'Ile-Verte, un étroit lambeau de terre, parcimonieusement découpé dans le vaste pays qui jadis leur appartenait tout entier." Taché, op. cit., p. 126-127.

23 Les Magouas, "c'étaient des fantômes protestants qui, la nuit venue, se transformaient en bêtes fabuleuses pour jeter la consternation chez les catholiques..." $(23,18)$ Quant aux Tarlanes, "ce sont de grandes créatures à tête de cheval qui, la nuit, surgissent dans les cimetières anglicans une fois que les catholiques en ont profané les tombes . Beaulieu, Docteur Ferron, p. 146. Cf. Jacques Ferron, La nuit, Montréal, Parti Pris, 1965, p. 88-90. 
les mythes et déformant les noms des héros helléniques, le digne sujet du "royaume des centaures" $(53,28)$ évoque le "talon d'Archille" $(20,27)$ et se plaît à raconter les péripéties d'Oscar, "le premier vrai homme-cheval de l'histoire" $(53,27)$, cet Icare nouvelle version qui, sur le dos d'un cheval ailé, tente de "monter jusqu'au soleil" $(10,34)$.

À l'opposé du conteur fantasque mais comme lui rattaché à l'univers fascinant de la Grèce antique, Philippe Couture entouré "de livres qui portent sur la tragédie" $(77,24)$ s'interroge et note: "Peut-être la fiction n'est-elle rien de plus que ce qui se meurt de tout événement pour devenir mémoire. Mais je n'en sais rien au fond. Pour le savoir, il me faudrait retourner à tous ces vieux auteurs grecs qui ont transgressé la mythologie pour nous donner la tragédie. " $(76,26$ et $77,24-25)$

La tragédie grecque ${ }^{24}$ puise en effet ses thèmes dans les épopées mythiques (tels la guerre de Troie, la destinée malheureuse d'CEdipe et de sa descendance). Mise en scène des mythes originels et de ses crimes monstrueux - parricides, fratricides, infanticides... -, elle s'attache d'autre part à la réalité collective de son époque. Doublement nourrie du passé mythique et de l'actualité politique, la tragédie les transcende néanmoins, transgresse l'un et l'autre par la visée interprétative qu'elle leur donne.

Nul besoin d'évoquer longuement l'influence déterminante de la tragédie grecque. Fleuron moderne de la tragédie, comme l'ont souligné certains de ses acteurs ${ }^{25}$, tel apparaît L'béritage

24 Jacqueline de Romilly, La tragédie grecque, Paris, Presses Universitaires de France, coll. "Littératures anciennes", 1970, p. 15 et passim.

25 À propos de Xavier Galarneau, le personnage qu'il incarne dans la série télévisée, le comédien Gilles Pelletier déclare: "C'est un homme possédé par l'amour, frappé par une malédiction... un peu comme Phèdre. [...] l'œuvre de Victor-Lévy Beaulieu ressemble plus au monde tragique d'Eschyle, de Sophocle, de Shakespeare, de Racine, de Claudel. " Ghislaine Rheault, "Gilles Pelletier compare "L'héritage" à une grande tragédie classique", dans Le Soleil, 13 décembre 1987, p. C-1. Un autre comédien, Yves Desgagnés alias Junior Galarneau, affirme pour sa part: "Les personnages de Victor-Lévy Beaulieu sont mythologiques. C'est une écriture qui remonte à l'origine. Les Grecs... Euripide... Eschyle. C'est directement inspiré de cela. Les familles incestueuses, des personnages porteurs d'une mission. Des personnages qui ne sont pas nés par hasard. Disons que c'est un voyage initiatique de personnages sur la terre." Raymond Bernachez, " "Les personnages de L'béritage de Victor-Lévy Beaulieu sont mythologiques" ", dans La Presse, 31 décembre 1988, p. D 2. 
avec ses guerres fratricides, ses relations incestueuses, de même que ses références à l'actualité ${ }^{26}$ et sa marche inexorable vers le destin. Sous la plume de l'écrivain, les conflits familiaux dépassent les frontières du pays et se colorent d'une portée universelle.

Lui-même poète et détenteur d'un vaste savoir livresque, Philippe Couture tient le rôle de choreute dans la tragédie téléromanesque. À l'instar du chœur, Couture occupe une place centrale. Il a «à intervenir, à supplier, à espérer [...] ses émotions scandent d'un bout à l'autre les diverses étapes de l'action" 27 . La même impuissance les caractérise: cette incapacité d'agir afin de contrer la moïra, le destin. Témoin et révélateur des forces surhumaines en jeu, Couture en appelle - comme Victor Hugo ${ }^{28}$ - à la "veine noire de la destinée " et à son implacabilité: "Peut-être la veine noire de la destinée interviendra-t-elle avant que tout ne soit joué " $(76,54)$; "Il n'y a rien à faire quand la veine noire de la destinée s'ameute dans la nuit " $(56,65)$.

Si la lecture prend un espace important dans le quotidien de certains personnages de L'béritage, il en est de même pour l'auteur. "Tout le temps qu'a duré L'béritage, confesse Victor-Lévy Beaulieu, je lisais la Bible ou bien Lao Tseu. Avant, c'était Aurobindo et la Bbagavad-gîtâ. Demain, ce sera peut-être Thérèse d'Avila ou saint Augustin " 29.

L'influence de ces lectures, celles notamment de la Bible, se fait singulièrement sentir avec les quelque cinquante extraits qui parsèment les diverses scènes du téléroman. Xavier, d'abord, utilise un curieux procédé pour lire la Bible: il "L'OUVRE AU HASARD ET, SANS REGARDER, POINTE UN PASSAGE DU

26 "Ça parlait du Québec d'aujourd'hui [...]. La mort du père, c'est la mort de René Lévesque. Les enfants qui se déchirent l'héritage, c'est nous autres. Et l'inceste, l'inceste entre Xavier et Miriam, c'est Lévesque qui voulait pas choisir, Lévesque qui aurait dû faire l'indépendance [...], Richard Martineau, "Flagrant délire", dans Voir, du 22 au 28 mars 1990, p. 10. Les propos de Victor-Lévy Beaulieu laissent entrevoir tout l'intérêt qu'il y aurait à faire une analyse socio-politique de L'béritage.

27 De Romilly, op. cit., p. 28.

28 "Nous avons beau tailler de notre mieux le bloc mystérieux dont notre vie est faite, la veine noire de la destinée y reparaît toujours. "Victor Hugo, Les misérables, Tome I, Paris, Nebon éditions, [s.d.], p. 298-299.

29 Victor-Lévy Beaulieu, Seigneur Léon Tolstoï. Essai-journal, Montréal, Stanké, 1992, p. 156. 
DOIGT 30 (cf. e.g., 4, 27). Le passage ainsi désigné aiguillonne ou conforte infailliblement le père dans ses décisions.

Loin de connaître les Écritures par cour comme Xavier (18, 85), Miville éprouve en plus quelques problèmes de compréhension. Relisant plus de dix fois le même extrait, il s'acharne à en déchiffrer le sens $(21,75)$. Il y a du moins une chose qui lui apparaît clairement: "Dans Bib'e, y a pas d'alliance véritab'e si y a pas un p'tit mouton pour la conclure." $(54,43)$ Ce qui l'amène à offrir un agneau à Rebecca Bloom, cette étudiante juive de Toronto, plus familière que son cavalier servant avec les textes sacrés. Leur passion s'achève dramatiquement puisque Rebecca, de passage à Trois-Pistoles, est au nombre des victimes de l'incendie de l'hôtel. Sarah Bloom sa mère, sorte de pendant féminin de Xavier, en appelle aussi à la loi divine: "Comme dit la Loi qui nous a permis de survivre, œil pour œil et dent pour dent. " $(82-30)$

Enfin, Moïse Abraham - ce petit-fils d'un ministre protestant à la recherche de ses origines et de l'amour de Miriam - , aux noms évocateurs, récite de longs passages bibliques. Citant Isaie ou professant à sa manière, les paroles revêtent un accent prophétique ${ }^{31}$ : "Nous tous, comme du petit bétail, nous étions errants... nous nous tournions chacun vers son chemin... et le Seigneur a fait retomber sur lui la perversité de nous tous." (Is. 53, 6) Tel Michée (Mich. 2, 1-3), Moïse annonce la malédiction: "Malheur à celui qui projette le méfait et qui manigance le mal! Malheur à celui qui se saisit de la maison et de l'héritage qui ne sont pas les siens! Que le Seigneur s'en empare et brûle jusqu'au dernier de ses os!» $(77,78)$ Inversant une parole de Jésus (Mt. 10, 34-36), Moïse proclame encore: "Je n'étais pas venu ici pour apporter le courroux mais la paix. Je n'étais pas venu ici pour apporter la guerre mais la sérénité. Car il y avait déjà trop longtemps que le soleil était tombé dans la mer. " $(84,61)$

30 Au fil des quatre-vingt-six épisodes, les indications scéniques de l'auteur sont inscrites en lettres capitales, afin de les distinguer des dialogues proprement dits.

31 Erzulie, qui l'a connu à une époque tourmentée de sa vie, lui rappelle: "prophète, c'est c'que tu prétendais êt'e à l'hôpital..." $(73,32)$ 
106

\section{Une sainte histoire}

À travers cette diversité des croyances qui s'entrecroisent, plusieurs pistes se dégagent. D'abord, sous les apparences d'une chronique familiale, L'béritage revêt des allures de (télé)roman national. La vision du monde qu'offre l'écrivain mène à une seconde piste, apparentée aux écrits vétéro et néotestamentaires. Enfin, si le vaudou, le bouddhisme et le paganisme notamment affleurent bien dans L'béritage, la tragédie hellénique, elle, occupe une place prépondérante.

L'béritage constitue d'abord une véritable histoire sainte du Québec, plus complexe et syncrétiste toutefois que celle à laquelle nous avons été habitués. Une histoire qui, à ses débuts, s'oriente principalement sur le chef des Galarneau, gardien des traditions familiales, Le protestantisme légaliste de Xavier n'est pas sans évoquer la rigidité du catholicisme propre au Québec de l'avantRévolution tranquille. La mort du père instaure une période de crise qui donne lieu à une croissance spirituelle tant individuelle que collective. Albertine et Junior par exemple entreprennent une quête de leurs origines et de leur identité. Avec l'entrée en scène de figures étrangères tels Éric, Erzulie et Rebecca, la tribu des Galarneau - sorte de microcosme québécois - s'ouvre progressivement au monde et à ses multiples cultures.

Ce déploiement du religieux culmine avec le sacrifice infernal de l'hôtel. La longue et chaotique recherche d'identité et la pénible guerre de succession engendrées par la mort du patriarche s'achèvent par cette tragique immolation. Lors de cette ultime catastrophe, c'est en effet le corps démembré du père, morcelé par les luttes fratricides, qui est enfin rassemblé par ses enfants ${ }^{32}$. "Aucun pays ne se fonde sans un sacrifice propitiatoire "33, note

32 Cette recomposition du corps paternel n'est pas sans évoquer celle du dieu égyptien Osiris qui, du souffle d'Isis et de Nephtys, ressuscite. ( $C f$. e.g., Christian Jacq, Pouvoir et sagesse selon l'Égypte ancienne, Monaco, Éditions du Rocher, 1981.) De même, créature des commencements, la figure hindoue Prajapati - à la fois divinité suprême, créateur et père des dieux - naît de l'amalgame de sept personnages engendrés par les souffles vitaux. ( $C f$. e.g., J. Varenne, Mythes et légendes extraits des Brâbamana, Paris, Gallimard, coll. "Connaissance de l'Orient", 1967, p. 27-29; Jan Gonda, Les religions de l'Inde, tome I, Paris, Payot, coll. "Bibliothèque historique", 1962, p. 227-230.

33 Victor-Lévy Beaulieu, Jacques Ferron conteur, émission présentée à la radio MF de Radio-Canada le 27 février 1992. 
en ce sens Beaulieu. La fiction dépasse ici le réel puisqu'à travers cette métaphore du corps paternel à nouveau recomposé, il s'agit de l'héritage, de la fondation d'un pays québécois.

Bien plus qu'il ne transcende la réalité nationale, l'univers imaginaire de Victor-Lévy Beaulieu invite les héritiers de cette terre longtemps promise à bâtir un ordre nouveau. Symbolisé par les funérailles des cinq victimes, ce n'est rien de moins que l'avènement de la réconciliation universelle, c'est-à-dire l'œcuménisme ${ }^{34}$ qui se réalise. De fait, l'espérance eschatologique, la fin de 1'histoire s'accomplit avec la réunion, dans un même temple, de gens de races et de cultes différents. De même, rabbin, prêtre catholique et ministre protestant officient côte à côte. Alors que s'ébranle le cortège funèbre, Miville observe: "Ça doit t'êt'e ça qu'on appelle la fin du monde. " $(84,70)$

À l'ancien monde succède un nouvel ordonnancement né du funeste événement de l'hôtel. Les unions ${ }^{35}$ qui se concluent entre les protagonistes se révèlent des indices de régénération et des signes d'une alliance neuve et solide. Sur la terre enfin habitée, le soleil se lève. L'astre souverain aux sempiternelles résurrections prend les allures du père comme dans les dessins de Miriam enfant où, figure surplombante, un "grand soleil souriant $[\ldots]$ fumait la pipe ainsi que le faisait alors Xavier"36. De plus, si Xavier - de l'arabe "brillant" - évoque l'éclat solaire, le soleil, lui, se nomme familièrement Galarneau. "Salut, Galarneau! Bonjour, Soleil! [...] C'est papa qui disait ça en se levant le matin. Il disait: notre père à tous c'est le soleil, il s'appelle Galarneau lui aussi, comme nous. Il nous regarde de là-haut, mais il est de la famille", écrit pour sa part Jacques Godbout ${ }^{37}$.

34 Le terme cecuménisme est utilisé ici dans sa signification première et non au sens restreint, qui a cours depuis le $x^{\mathrm{e}}$ siècle, de l'union de la Chrétienté. "Étymologiquement, ces mots [œcuménisme et œcuménique] viennent d'une expression grecque qui veut dire "la terre habitée" [...] la terre entière . Berthe Gavalda, Le mouvement cecumënique, Paris, Fresses univerversitaires de France, coll. "Que sais-je?", no 841, 1959, p. 5.

35 Mariages et contrats divers apparaissent en grand nombre dans le dernier épisode.

36 Beaulieu, L'béritage I, p. 150.

37 Jacques Godbout, Salut Galarneau!, Paris, Seuil, 1967, p. 57. "Galarneau, ça veut dire soleil en français..." $(51,32)$, s'exclame pour sa part Rebecca Bloom.) Mais si, comme le rappelle Godbout, le soleil "s'appelle Galarneau", ce patronyme signifie également "pluie froide. Cf. N. E. Dionne, Les Canadiens français. Origines des familles émigrées de 
108

$$
*_{* *}^{* *}
$$

Histoire sainte du Québec, disions-nous précédemment de L'béritage. Cela s'avère d'autant plus juste que le téléroman reprend plusieurs thèmes de l'Ancien Testament. L'un et l'autre relatent globalement les tribulations d'un peuple élu. Il s'agit somme toute de la longue marche des Galarneau et des juifs vers la terre promise, en attente de la libération. Des faits communs s'annoncent dès la genèse des deux récits. La chute hors du paradis est, par exemple, l'œuvre d'un couple fautif. Pour n'avoir su résister à la tentation, Adam et Ėve sont chassés du jardin d'Éden. L'univers de Xavier et de sa fille bascule également. Leur union incestueuse marque la perte de l'état d'innocence, l'exil pour Miriam et la fin de l'ère de prospérité pour l'homme de chevaux. Beaulieu emploie d'ailleurs l'expression "faute originelle" pour qualifier la nuit fatale du couple au château Frontenac ${ }^{38}$.

Dès les commencements de l'épopée beaulieusienne, les héros doivent subir "quasiment un déluge" $(1,20)$ pendant plus d'une semaine $(2,2)$. Ces précipitations, dévastatrices pour les cultures, ne sont pas sans évoquer les pluies diluviennes que la colère de Yahvé déclenche durant quarante jours (Gen. 7,4 ) contre l'humanité pécheresse. Un autre déluge en revanche, de neige celui-là, ponctue l'expulsion d'Albertine l'infidèle: une "tempête qui a tout emporté dans ce désordre qu'est l'hiver" 39 . Devant l'ensevelissement de la nature pour quelque six mois dans un désert de neige, les protagonistes n'ont d'autre recours que de se retirer dans leur maison, tel Noé dans l'arche. La longue gestation qu'impose l'interminable hiver québécois évoque, toutes proportions gardées, le séjour quarantenaire des Hébreux au désert.

La correspondance établie ici entre le peuple choisi des écrits vétérotestamentaires et celui du Québec - représenté en microcosme par le clan Galarneau - a de profondes racines. La survie providentielle des colons français du Nouveau Monde rappelle

France, d'Espagne, de Suisse, etc., pour venir se fixer au Canada, depuis la fondation de Québec jusqu'à ces derniers temps et signification de leurs noms, Québec, Laflamme \& Proulx imp., 1914, p. 280.

Ibid., p. 56. 
celle des Hébreux. Les origines même de la nation baignent dans une aura mystique qu'hagiographes et historiens du Québec ont longtemps entretenue. À ce propos, Serge Gagnon 40 écrit:

Pour la grande majorité des historiens, c'est Dieu qui est l'agent principal du développement historique. Il fait échouer les tentatives de colonisation du xvi ${ }^{\mathrm{e}}$ siècle parce que les immigrants n'ont pas les vertus chrétiennes nécessaires à la naissance du peuple choisi par Dieu. [...] Les révolutions américaine et française, poursuit Gagnon, donnent naissance à des sociétés sans Dieu. Le peuple élu va devenir le gardien de la vérité en Amérique.

"Y a rien qui était innocent, dans L'béritage, affirme Beaulieu lors d'une interview. Y a rien qui était là par hasard... Ça parlait du Québec d'aujourd'hui, du passage de l'Ancien au Nouveau Testament, de la prise de pouvoir par les femmes, bref, d'un paquet d'affaires." 41 Une courte scène du téléroman illustre éloquemment la transition de l'un à l'autre Testament et l'intronisation de la femme:

MIRIAM REFERME LA PORTE, VA VERS LE SECRÉTAIRE. ELLE S'ASSOIT DANS LE FAUTEUIL DE XAVIER ET OUVRE LA BIBLE AU HASARD. ON VOIT LA PAGE DE GAUCHE QUI EST BLANCHE, PUIS CELLE DE DROITE SUR LAQUELLE ON PEUT LIRE: NOUVEAU TESTAMENT. (41, 43-44)

Quelques autres thématiques propres aux écrits vétéro et néotestamentaires se rencontrent encore dans L'béritage. Promesses et alliances jalonnent les divers récits. Les actions extraordinaires, indices de la miséricorde divine, parsèment également la longue marche eschatologique. Aux guérisons miraculeuses qu'opèrent Miriam ou Julie à l'endroit de Xavier ou de Maxime (24, 8 et $73 ; 47,46-47)$ répondent les prodiges accomplis par Moïse notamment lors de l'Exode et, plus particulièrement, les pouvoirs de thaumaturge de Jésus. À l'instar de nombreux personnages bibliques visités en songe par des créatures célestes ou soumis, lors de leur sommeil, à d'étonnantes prémonitions, le rêve tient en outre une grande place chez plusieurs héros du téléroman. C'est

40 Serge Gagnon, Le Québec et ses bistoriens de 1840 à 1920. La NouvelleFrance de Garneau à Groulx, Québec, Presses de l'Université Laval, coll. "Les cahiers d'histoire de l'Université Laval", no 23, 1978, p. 416417.

41 Martineau, loc. cit., p. 10. 
110

ainsi qu'un cauchemar aux signes prophétiques décide du retour du fils puîné auprès de son père agonisant $(37,25)$. Au cours d'un rêve prémonitoire, Julie la benjamine flotte dans les airs avec Junior; tous deux munis de "grandes ailes blanches", ils survolent Trois-Pistoles d'où l'hôtel a disparu $(81,77-78)$. Les voies du divin sont impénétrables, dit-on, enclines à des revirements imprévus, à des solutions inédites. Cette prédilection pour le cadet au détriment de l'aîné, par exemple, est présente avec l'élection de Junior le marginal à titre de "fils bien-aimé" $(38,66)$. L'héritier présomptif, Miville, se voit continuellement évincé, bafoué, contraint au rôle de bouc émissaire (e.g. 38, 7). Dans la Bible, le statut d'aîné semble par ailleurs fortement empreint de fatalité. Afin de commémorer l'exode marqué de fait par l'extermination des premiers-nés (Ex. 12, 29-34), la loi de Moïse prescrit que "tout premier-né mâle sera consacré au Seigneur" (Ex. 13, 12-13; Lc 2, 23). Le Christ, lui-même premier-né, se substitue à l'agneau pascal lors de la dernière Cène.

Le sacrifice apocalyptique - c'est-à-dire, au sens étymologique, celui qui révèle - de l'hôtel et le rassemblement œecuménique, dont il a été précédemment question, présentent à leur manière la Parousie. Ils annoncent l'accomplissement de l'histoire: "Alors viendra la fin" (Mt 24, 14), l'atteinte du royaume promis et la réconciliation cosmique.

Le festin de noces du dernier épisode illustre magistralement cette ultime communion. Avec la réconciliation fraternelle, c'est par ailleurs l'être tout entier du père qui ressuscite, pour qu'apparaisse enfin le sens de l'histoire et la vérité de chacun ${ }^{42}$.

Si L'béritage puise substantiellement aux sources bibliques, les dernières paroles que prononce Miriam, avant de reprendre la route, sont pour leur part empreintes de l'esprit hellénique. "On comprend seul'ment quand on reste à sa place ou ben qu'on la r'trouve. Sinon, n'importe quel héritage, c'comme du sable mouvant: on fait rien d'plusse que s'enliser d'dans. " $(86,78)$ Après les tentatives d'usurpation, les conflits fratricides auxquels se sont livrés les protagonistes, la thémis ('ordre établi) est enfin reconquise. À l'hybris, la démesure, inéluctablement châtiée, succède

42 Comme dans la tragédie grecque, "c'est au terme du drame que les actes prennent leur vraie signification et que les agents découvrent, à travars ce qu'ils ont réellement accompli sans le savoir, leur vrai visage". (JeanPierre Vernant et Pierre Vidal-Naquet, Mythe et tragédie en Grèce ancienne 1, Paris, La Découverte, 1986 [1972], p. 37-38.) 
pour chacun un retour à l'espace qui lui est réservé dans l'ordre cosmique.

Mosaïque de croyances les plus diverses, le téléroman de Victor-Lévy Beaulieu dessine, en filigrane, une véritable histoire sainte aux accents tragiques. De la quête forcenée de l'héritage, des errances de ces personnages plus grands que nature que sont les Galarneau - destinés par leur patronyme à faire la pluie et le beau temps - naît le rêve. Leur advient alors au fil des mots la souveraineté, l'avènement d'une "terre habitée". Toutefois, comme chez les Tragiques, la conciliation, "la victoire sur toutes les forces du passé ont moins le caractère d'un constat, d'une tranquille assurance, que d'un espoir et d'un appel, où l'angoisse ne cesse jamais d'être présente, même dans la joie des apothéoses finales " 43 .

"Après, dira encore Miriam, ça s'ra une aut'e histoire... Après, c'toujours une aut'e histoire." $(86,81)$ Immuablement, au-dessus du fleuve, Galarneau s'élève... 